\title{
CRITICAL CONDITIONS FOR CARBON COMBUSTION
}

\author{
C. TREVIÑO \\ Div. Ing. Mec. Eléct., Div. Est. Posgrado \\ Facultad de Ingeniería, LNAM \\ 04510 México, D.F., MEXICO \\ F. HIGUERA AND LIÑÁ. \\ E.T.S.I. Aeronáuticos, UPM \\ Plaza del Cardenal Cisneros 3 \\ Madrid, SPAIN
}

\begin{abstract}
The critical conditions for vigorous burning of spherical carbon particles are derived using asymptotic techniques for large Zeldovich numbers. The particle lifetime and the temperature history are obtained for both supercritical and subcritical regimes, when a carbon particle is suddenly exposed to a stagnant high temperature oxidizing atmosphere. For Damköhler numbers below a critical number, that is, the subcritical regime, slow burning occurs in the carbon particle. That means that throughout the life of the particle, the chemical reaction is controlled by kinetics. In this regime, the lifetime is extremely long. On the other hand, for Damköhler numbers above the critical one, the process ends with a rapid increase of temperature finally reaching a reaction controlled by diffusion. In this supercritical regime, the life is relatively short. For values of the reduced Damköhler number very close to the critical one, a Ricatti equation is derived for the non-dimensional temperature of the particle, the solution of which defines whether the particle undergoes subcritical or supercritical behavior.
\end{abstract}

\section{Introduction}

The combustion of carbon has received special attention in the literature in the past vears. To study the interaction of the flow field with the carbon combustion, several flow geometries have been selected. The stagnationflow geometry has been used for both experimental and theoretical works. ${ }^{1-5}$ The principal objectives in most of these works have been the study of different regimes in carbon combustion and the interaction of homogeneous and heterogeneous reactions with the flow field. The influence on carbon combustion of the water vapor content of the atmosphere, has also been evaluated. ${ }^{3,4}$ The combustion of spherical carbon particles has been the object of several studies. ${ }^{6-12}$ Ubhayakar $^{6}$ studied the quasisteady burning and extinction of spherical particles (carbon) in a quiescent oxidizer gas. Libby et al. ${ }^{7,8,10,11}$ analyzed the steady-state and transient combustion of carbon particles suddenly inmersed in a hot oxidizing atmosphere. In this context, the effect of the atmospheric water vapor concentration was also evaluated. ${ }^{10}$ In the last work of the series, Kassoy and Libby ${ }^{11}$ used high activation asymptotic methods for high Zel'dovich numbers of the hetero- geneous reaction $\left(2 \mathrm{C}+\mathrm{O}_{2} \rightarrow 2 \mathrm{CO}\right.$ ), in order to study the entire history of a carbon particle. They analyzed different regimes and compared the results with the numerical solution of the governing equations. They found very good agreement for critical temperatures not too close to the ambient temperature. The analysis of Kassoy and Libby fails when the temperatures are close to each other.

The objective of this paper is to extend the analysis of Kassoy and Libby ${ }^{11}$ to the case where the critical temperature is close to the high ambient temperature. It is in this regime that we obtain the critical conditions needed for a carbon particle to achieve vigorous heterogeneous combustion. We show that activation energy asymptotic methods are well suited to the study of the transient behavior in this limiting case.

\section{Formulation}

We consider the following physical problem. A spherical carbon particle of radius $R_{0}$ is suddenly injected in a hot oxidizing atmosphere of temperature $T_{x}$ and oxygen concentration $Y_{02 \times}$. Only the direct heterogeneous 
reaction $2 \mathrm{C}(s)+\mathrm{O}_{2} \rightarrow 2 \mathrm{CO}$, is assumed, the reation rate of which is given by the Arrhenius law:

$$
r=B Y_{\mathrm{O}_{2}} \exp \left(-\frac{E}{\bar{R} T_{s}}\right)
$$

where $B$ is the pre-exponential factor; $E$ is the activation energy; $T_{s}$ corresponds to the temperature at the solid/gas interface and $\bar{R}$ is the universal gas constant. Assuming quasi-steady behavior in the gas phase, the governing equations are given by:

Gas phase

$$
\begin{gathered}
m R^{2} C_{p} \frac{d T}{d r}=\frac{d}{d r}\left(\lambda_{g} r^{2} \frac{d T}{d r}\right) \\
m R^{2} \frac{d Y_{i}}{d r}=\frac{d}{d r}\left(\rho D_{i} r^{2} \frac{d Y_{i}}{d r}\right), \text { for } i=\mathrm{O}_{2}, \mathrm{CO}
\end{gathered}
$$

Solid phase

$$
\rho_{s} C_{s} \frac{\partial T}{\partial_{t}}=\frac{\lambda_{s}}{r^{2}} \frac{\partial}{\partial r}\left(r^{2} \frac{\partial T}{\partial r}\right)
$$

with the boundary conditions given as

$$
\begin{gathered}
\text { at } r \rightarrow \infty, \quad T=T_{\infty}, \quad Y_{i}=Y_{i \infty} \\
\text { at } r=0, \quad \frac{\partial T}{\partial r}=0 \\
\text { at } \left.r=R, \quad \lambda_{g} \frac{d T}{d r}\right)_{g}=-m Q+m T_{s}\left(C_{p}-C_{s}\right) \\
\left.\quad+J\left(T_{s}^{4}-T_{\infty}^{4}\right)+\lambda_{s} \frac{\partial T_{s}}{\partial r}\right)_{s} \\
m=W_{c} r=W_{c} B Y_{\mathrm{O}_{2}} \exp \left(-\frac{E}{\bar{R} T_{s}}\right) \\
\left.\rho D_{1} \frac{d Y_{\mathrm{O}_{2}}}{d r}\right)_{g}=m\left(Y_{\mathrm{O}_{2}}+\frac{W_{\mathrm{O}_{2}}}{2 W_{c}}\right)
\end{gathered}
$$

The initial condition is the following:

$$
T=T_{s} \text { at } t \leq 0 \text { for } r \leq R_{0}
$$

The instantaneous radius of the particle is changing at a rate given by

$$
\frac{d R}{d t}=-\frac{m}{\rho_{s}}
$$

The meaning of the variables are described in the nomenclature. The quasi-steady behavior in the gas phase is fully justified because the ratio of the characteristic time in the gas $\left(t_{g}\right)$ to that of the solid $\left(t_{s}\right)$ is given by

$$
\frac{t_{g}}{t_{s}}-\frac{\rho_{g} C_{p} \lambda_{s}}{\rho_{s} C_{s} \lambda_{g}} \ll 1
$$

This ratio is much less than unity due mainly to the density ratio. Due to the relatively high thermal conductivity of the carbon in comparison with that of the air, a uniform temperature of the solid is also assumed. This would change, of course, if the particle was subjected to forced or free convection. The equations in the gas phase are readily integrated:

$$
\begin{aligned}
T= & \frac{T_{s}-T_{\infty} \exp (-\beta)}{1-\exp (-\beta)} \\
& +\frac{\left(T_{\infty}-T_{s}\right)}{1-\exp (-\beta)} \exp \left(-\frac{\beta R}{r}\right)
\end{aligned}
$$

and

$$
\begin{aligned}
Y_{i}= & \frac{Y_{i s}-Y_{i \infty} \exp (-\beta \mathrm{Le})}{1-\exp (-\beta \mathrm{Le})} \\
& +\frac{\left(Y_{i \infty}-Y_{i s}\right)}{1-\exp (-\beta \mathrm{Le})} \exp \left(-\frac{\beta \mathrm{Le} R}{r}\right)
\end{aligned}
$$

where $\beta=m R C_{p} / \lambda_{g}$ is the non-dimensional mass flow rate and $L e=\lambda_{g} / \rho D C_{p}$ is the Lewis number which can be assumed to be the same for all the species. Assuming a uniform temperature in the solid, the heat flux at the solid/gas interface is given by

$$
\left.\lambda_{s} \frac{\partial T}{\partial r}\right)_{s}=\frac{R \rho_{s} C_{s}}{3} \frac{d T_{s}}{d t}
$$

Introducing the following non-dimensional variables

$$
\begin{aligned}
& \Theta=\frac{T_{\infty}-T_{s}}{T_{\infty}-T_{s}{ }^{\circ}} \\
& y=\frac{Y_{\mathrm{O}_{2}}}{Y_{\mathrm{O}_{2} \infty}} \\
& \tau=\frac{R_{0}^{2} \rho_{s} C_{p}}{\lambda g} \\
& a=\frac{R}{R_{0}}
\end{aligned}
$$

the non-dimensional gas solid interface relations transform to 


$$
\begin{aligned}
& \frac{\beta \Theta}{\exp (\beta)-1}=-\beta\left[Q^{*}+\left(1-\frac{C_{s}}{C_{p}}\right) \Theta\right] \\
&+\bar{J} a\left[(1-\alpha \Theta)^{4}-1\right]-I a^{2} \frac{d \Theta}{d t} \\
& \frac{1-y}{\exp (\beta \text { Le })-1}=y+\vec{\nu} \\
& \beta= a \Delta y \exp \left[-\frac{\alpha \Theta}{\epsilon(1-\alpha \Theta)}\right] \\
& a \frac{d a}{d \tau}=-\beta
\end{aligned}
$$

where the resulting non-dimensional parameters are defined as:

$$
\begin{aligned}
& Q^{*}=\frac{1}{\alpha}\left[\frac{Q}{C_{p} T_{\infty}}+\frac{C_{p}-C_{s}}{C_{p}}\right] \\
& \bar{J}=\frac{J R_{0} T_{\infty}^{4}}{\left(T_{\infty}-T_{s}\right) \lambda_{g}} \\
& \alpha=\frac{T_{\infty}-T_{s \infty}}{T_{\infty}} \\
& I=\frac{C_{s}}{3 C_{p}} \\
& \bar{\nu}=\frac{4}{3 Y_{\mathrm{O}_{2} \infty}} \\
& \Delta=\frac{W_{C} B Y_{\mathrm{O}_{2} \infty} R_{0} C_{p}}{\lambda_{g}} \exp \left[-\frac{E}{\bar{R} T_{\infty}}\right] \\
& \epsilon=\frac{\bar{R} T_{\infty}}{E}
\end{aligned}
$$

The parameters listed in the above lines are of order of unity except $\Delta$ and $\epsilon \cdot \Delta$ is the Damköhler number and can be much larger or much smaller than unity. $\varepsilon$ is the inverse of the non-dimensional activation temperature, and for this case is a small number. The initial condition is given by

$$
\Theta(0)=1
$$

We define a critical non-dimensional temperature $\theta_{c}$, which satisfies the transcendental relation

$$
\Theta_{c}+\frac{\epsilon}{\alpha} \Theta_{c}=\frac{\epsilon}{\alpha} \ln \Delta
$$

Therefore, the non-dimensional mass burning rate $\beta$ is given by

$$
\beta=a y \Theta_{c} \exp \left[\frac{\alpha}{\epsilon} \frac{\left(\Theta^{*}-\Theta\right)}{\left(1-\alpha \Theta^{*}\right)(1-\alpha \Theta)}\right]
$$

where

$$
\Theta^{*}=\frac{\Theta_{\varepsilon}}{1+\alpha \Theta_{c}}
$$

For values of $\Theta$ larger than the critical value, $\theta^{*}, \beta$ is exponentially small and thus, the process is chemically frozen. As $\theta$ approaches $\Theta^{*}$, the chemical reaction is important and has to be taken into account. There are two distinct limits in this problem. For values of $\theta^{*} \gg \epsilon$, that is, for values of the Damköhler number $\Delta \gg 1$, there exists first a warm up stage where $\theta$ decreases from 1 to $\theta^{*}$. It is followed by a very short transition stage where the chemical reaction changes from kinetic to diffusion controlled, until the particle is virtually consumed. This limit has been analyzed by Kassoy and Libby. ${ }^{11}$ However, for values of the critical non-dimensional temperature $\Theta^{*}=\theta_{\text {c }}$ of order $\epsilon$, the response of the particle changes abruptly. This important limit is analyzed in the next section.

\section{Analysis}

As mentioned earlier, for values of the nondimensional temperature of the particle $\theta$ larger than $\theta_{c}$ (which is assumed in this distinct limit to be of order $\varepsilon$ ), the variables $\beta, d a / d \tau$ and $(1-y)$ are exponentially small. In this inert stage, $\theta$ decreases from unity reaching asymptotically the value of zero as $\tau \rightarrow \infty$. However, when $\theta \sim \theta_{c} \sim \epsilon$, the chemical reaction is important in reaching a transition stage. For a concrete critical value of $\Delta, \Delta_{c}$, the critical conditions are obtained. For $\Delta<\Delta_{\text {c }}$ (subcritical regime), slow burning occurs in the carbon particle. That means that during the whole life of the particle, the chemical reaction is controlled by kinetics. On the other hand, for values of $\Delta>\Delta_{c}$, the transition stage is very long (of the same order of magnitude as the inert stage) and ends with a rapid increase of the temperature, reaching the diffusion-controlled regime. The different stages are analyzed below.

Inert stage. For values of the non-dimensional temperature of the particle such as $\theta>\theta$, the chemical reaction is exponentially small. This means that the oxygen concentration at the carbon surface can be assumed to be the same as the ambient one. For the same reason, the particle radius does not change in this inert stage. The gas/solid interface relations transform to 


$$
I \frac{d \Theta_{f}}{d t}=\bar{J}\left[\left(1-\alpha \Theta_{f}\right)^{4}-1\right]-\Theta_{f}
$$

with the initial condition $\theta_{f}(0)=1$. The subscript $f$ denotes the temperature for this inert stage. Equation (18) with the initial condition can be written as

$$
\int_{1}^{\Theta} \frac{d \Theta_{f}}{\bar{J}\left[\left(1-\alpha \Theta_{f}\right)^{4}-1\right]-\Theta_{f}}=\tau
$$

The asymptotic behavior for $\theta_{f}$ as $\tau \rightarrow \infty$ is given by

$$
\Theta_{f} \sim c \exp \left|-\left(\frac{1+4 \bar{J} \alpha}{I}\right) \tau\right|
$$

where $c$, of order unity, is a function of $\alpha, \bar{J}$ and I. If we define $\tau^{*}$ as the time model for the particle to reach the non-dimensional temperature $\theta_{f}$ the value of $\epsilon$, then

$$
\Theta_{f} \sim \epsilon \exp \left[-\left(\frac{1+4 \vec{J} \alpha}{I}\right) \sigma\right]
$$

with

$$
\tau^{*} \approx-\left(\frac{I}{1+4 \bar{J} \alpha}\right) \ln \epsilon
$$

and

$$
\sigma=\tau-\tau^{*}
$$

Transition stage As $\Theta$ approaches a value of order $\varepsilon$, the chemical reaction is important and has to be retained in this transition stage. In this case, it is convenient to define

$$
\theta=-\frac{\epsilon}{\alpha} \phi
$$

with $\phi$ of order unity. Introduction of this relation into Eqs. (11) through (14) gives

$$
\begin{gathered}
a^{2} \frac{d \phi}{d \bar{\sigma}}=-\left[\frac{\beta}{\exp (\beta)-1}+\tilde{J} a\right] \frac{\phi}{1+\tilde{J}} \\
+a y \delta \exp (\phi) \\
\frac{1-y}{\exp (\beta \operatorname{Le})-1}=y+\bar{\nu} \\
\beta=\frac{a(1-\tilde{J}) \delta y \exp (\phi)}{Z} \\
\frac{d a}{d \bar{\sigma}}=-\frac{\delta y I}{Z} \exp (\phi)
\end{gathered}
$$

with

$$
\begin{aligned}
& \tilde{J}=4 \bar{J} \alpha \\
& Z=\frac{Q^{*} \alpha}{\epsilon} \\
& \delta=\frac{\Delta Z}{(1+\tilde{J})} \\
& \bar{\sigma}=\left(\frac{1+\tilde{J}}{I}\right) \sigma
\end{aligned}
$$

Here, $Z$ denotes the appropriate Zel'dovich number or the ratio of the activation energy of the surface reaction to the thermal energy. $\delta$ represents the reduced Damköhler number and is of the order of unity in this limit. Matching with the inert stage is achieved through

$$
\phi \sim \alpha \exp (-\bar{\sigma}) \text { as } \bar{\sigma} \rightarrow-\infty
$$

In the limiting case of $Z \rightarrow \infty$, Eqs. (23) through (26) reduce to

$$
\frac{d \phi}{d \bar{\sigma}}=-\phi+\delta \exp (\phi)
$$

with the initial condition given by the matching condition (27). Equation (28) is the classical one, resulting in thermal explosion problems. For $\delta$ $>e^{-1}, \phi \rightarrow \infty$ at a well defined non-dimensional time $\overline{\boldsymbol{\sigma}}_{I}$. On the other hand, for $\delta<e^{-1}$, $\phi$ tends to a pseudo-steady state given by the lower branch solution of the transcendental equation

$$
\phi_{e}=\delta \exp \left(\phi_{e}\right)
$$

As is well known, there are two values of $\phi_{e}$ for each value of $\delta$ below a critical one, and no solution for larger values of $\delta$. Only the lowest branch has physical meaning in this context. Figure 1 shows the non-dimensional temperature $\phi$ as a function of the non-dimensional time $\bar{\sigma}$ for three different values of $\delta$. Figure 2 shows the critical non-dimensional time needed to obtain a runaway reaction, as a function of $\delta$.

Subcritical regime. For $\phi<e^{-1}$, there are two characteristic time scales which define two different stages. In the first one the evolution of $\phi$ is governed by Eq. (28). Here $\phi \rightarrow \phi_{e}$ as $\bar{\sigma}$ $\rightarrow \infty$. In this pseudo steady state, $\beta$ of order $\varepsilon$ is small but different from zero. It is followed by a second slow stage, where the radius of the particle is governed by equation (26) in the form 


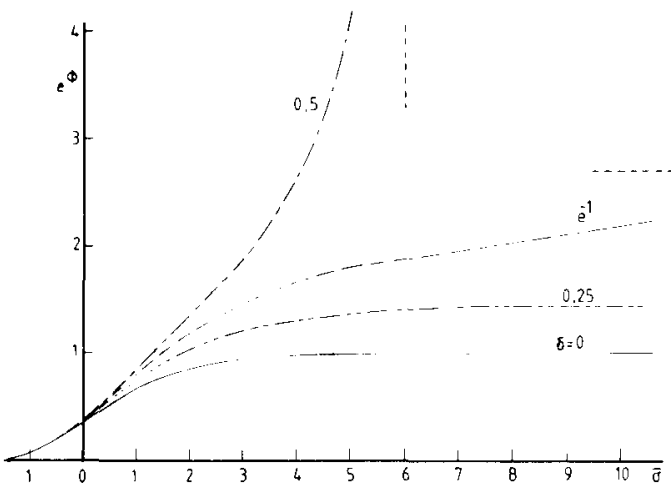

Fig. 1. Non-dimensional temperature $\phi$ history of the carbon particle during the transition stage for three different values of $\delta$.

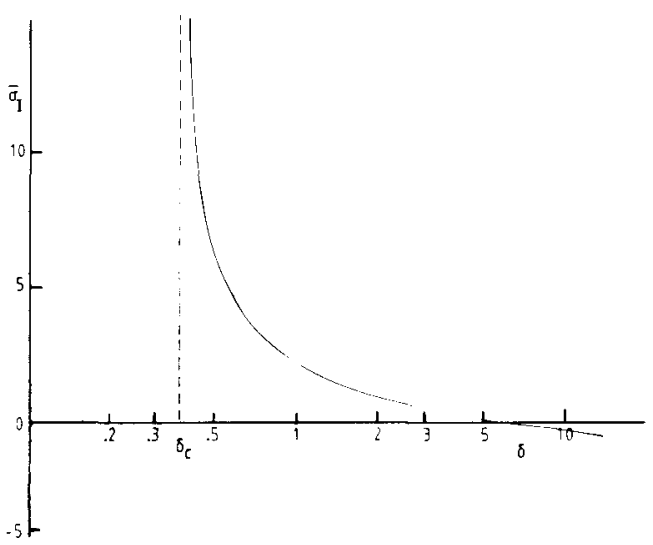

Fig. 2. Critical non-dimensional time $\bar{\sigma}_{l}$ in the transition stage needed to reach the diffusion controlled stage as a function of $\delta$.

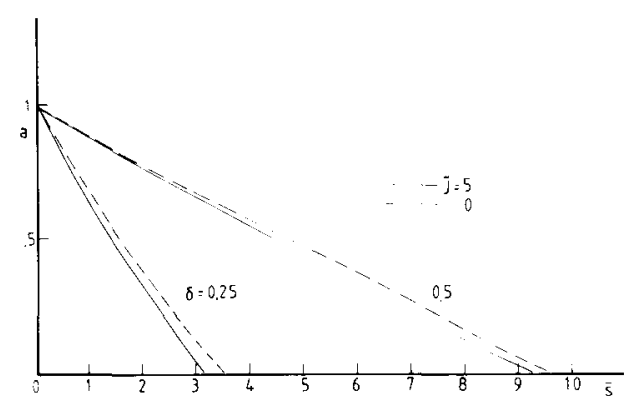

Fig. 3. History of the non-dimensional particle radius $a$ in the sub-critical regime for different values of $\bar{j}$ and $\delta$.

$$
\frac{d a}{d \sigma^{\prime}}=-\delta \exp \left(\phi_{e}\right)
$$

where

$$
\sigma^{\prime}=\frac{I}{Z} \bar{\sigma}
$$

In this slow time scale, the radius of the particle decreases because of the small mass burning rate B. In Eq. (30), $\tau<_{\varepsilon}$ satisfies the quasi-steady state governed now by the following relationship:

$$
\left(\frac{1+\tilde{J} a}{1+\tilde{J}}\right) \phi_{\rho}=\delta_{a} \exp \left(\phi_{e}\right)
$$

with the initial condition $a(0)=1$. In Figure 3 the evolution of " $a$ " with the time, is plotted for different values of $\tilde{J}$ and $\delta$.

Supercritical regime. For $\delta>e^{-1}, \phi \rightarrow \infty$ at a well defined critical value of $\bar{\sigma}_{I}$. At the end of this transition stage, $y$ falls to zero and $\beta$ reaches the diffusion controlled value of

$$
\beta=\beta_{d}=\frac{\ln \left(1+\frac{1}{\nu}\right)}{\text { Le }}
$$

In order to study the further evolution of the particle radius and temperature, it is convenient to return to the variable $\theta$ and to define a new non-dimensional time

$$
\tilde{\tau}=\tau-\tau_{I}
$$

where

$$
\tau_{I}=\left(\frac{I}{1+\tilde{J}}\right)\left(\bar{\sigma}_{I}-\ln \epsilon\right)
$$

Therefore, the interface relations take the form

$$
\begin{gathered}
\frac{\beta_{d}}{\exp \left(\beta_{d}\right)-1}=-\beta_{d}\left[Q^{*}+\left(1-\frac{C_{s}}{C_{p}}\right) \Theta\right] \\
\left.+\bar{J} a\left[(1-\alpha \Theta)^{4}-1\right)\right]-I a^{2} \frac{d \Theta}{d \tau} \\
a \frac{d a}{d \bar{\tau}}=-\beta_{d}
\end{gathered}
$$

with the initial conditions given by

$$
\begin{aligned}
& a(0)=1 \\
& \Theta(0)=0
\end{aligned}
$$

Both Eqs. (35) and (36) are autonomous and can be reduced to a single equation with the phase-space variables $(\Theta, a)$ in the form 


$$
\begin{aligned}
I \frac{d \Theta}{d a}= & \frac{\Theta}{a\left[\exp \left(\beta_{d}\right)-1\right]}+\frac{1}{a}\left[Q^{*}+\left(1-\frac{C_{s}}{C_{p}}\right) \Theta\right] \\
& -\bar{J}\left[(1-\alpha \Theta)^{4}-1\right]
\end{aligned}
$$

with the initial condition $\Theta(1)=0$.

The asymptotic behavior for small $a$ can be obtained from Eq. (37) as

$$
\Theta--\frac{Q^{*}}{K}+(c a)^{K / I} \text { for } a \rightarrow 0
$$

where

$$
K=\left[\frac{1}{\exp \left(\beta_{d}\right)-1}+1-\frac{C_{s}}{C_{p}}\right]
$$

and $c$ is the integration constant to be obtained from the solution of the exact Eq. (37). In fact, Eq. (38) gives the exact solution, neglecting radiation $(J=0)$. In this case,

$$
c=\left(\frac{Q^{*}}{K}\right)^{l / K}
$$

Therefore, Eq. (38) takes the form

$$
\Theta=\frac{Q^{*}}{K}\left(a^{K / I}-1\right)
$$

The lifetime of the carbon particle for this supercritical regime is then given by

$$
\tau_{\ell}=\left(\frac{I}{1+\tilde{J}}\right)\left(\bar{\sigma}_{I}-\ln \epsilon\right)+\frac{1}{2 \beta_{d}}
$$

Equation (39) can be rewritten, as a function of $\bar{\tau}$, in the form which gives the non-dimensional temperature of the particle as a function of the non-dimensional time for this diffusion limited stage:

$$
\Theta=\frac{Q^{*}}{K}\left(\left(1-2 \beta_{d} \bar{\tau}\right)^{K / 2 I}-1\right)
$$

Critical regime. For values of $\delta$ close to the critical value $\delta_{c}$, there are two different processes for this transition stage. In the first one, for values of $\bar{\sigma}$ of order unity, $\phi$ increases to values close to the critical value, which in first order is equal to one. It is followed by a very slow process, which depending on the value of $\delta$, either reaches a thermal runaway at a well defined time (supercritical) or $\phi$ reaches a maximum and then decreases with time, reaching asymptotically the subcritical regime. To analyze this regime, it is convenient to introduce the following expansions

$$
\begin{gathered}
\delta=\delta_{c}\left(1+\epsilon^{2 / 3} d+\ldots\right) \\
\phi=1+\epsilon^{1 / 3} \phi_{1}+\epsilon^{2 / 3} \phi_{2}+\ldots
\end{gathered}
$$

From Eqs. (23) to (26), it can be shown that the ignition time for this case is of order $\epsilon^{-1 / 3}$. Based on this fact, it is also convenient to introduce a new time scale of the form

$$
s=\epsilon^{1 / 3} \bar{\sigma}
$$

In this regime, $\beta$ can be evaluated from the critical conditions and is given by

$$
\beta=\frac{(1+\tilde{J})}{Q^{*} \alpha} \epsilon
$$

in a first approximation. In the same form, from Eq. (24), we obtain after expanding the exponential term

$$
y=1-\frac{(1+\tilde{J})}{Q^{*} \alpha} \operatorname{Le}(1+\bar{\nu}) \epsilon
$$

Introducing Eqs. (44) through (46) into Eq. (26), the particle radius is given by

$$
a=1-\frac{I s \epsilon^{2 / 3}}{Q^{*} \alpha}
$$

Equation (47) shows that the particle radius decreases linearly with time in this regime. Introducing the relations (42) through (47) into the Eq. (23), we obtain a Ricatti equation for $\phi_{1}$ in the form

$$
\frac{d \phi_{1}}{d s}=d-\frac{s}{(1+\tilde{J}) Q^{*} \alpha}+\frac{\phi_{1}^{2}}{2}
$$

which can be reduced further to

$$
\frac{d \omega}{d \bar{s}}=-\bar{s}+\omega^{2}
$$

with

$$
\begin{aligned}
\phi_{1} & =\Lambda \omega \\
\bar{s} & =\frac{\Lambda}{2}\left[s-d(1+\tilde{J}) Q^{*} \alpha\right] \\
\Lambda & =\left[\frac{4}{(1+\tilde{J}) Q^{*} \alpha}\right]^{1 / 3}
\end{aligned}
$$

The initial condition is given by

$$
\omega \rightarrow-\infty \text { for } \bar{s}=-\bar{d}=-\frac{\Lambda}{2}(1+\tilde{J}) Q^{*} \alpha d
$$

The associated second order linear differential equation to the Ricatti Eq. (49), is the Airy equation 


$$
\frac{d^{2} u}{d \bar{s}^{2}}-\bar{s} u=0
$$

where

$$
\omega=-\frac{1}{u} \frac{d u}{d \bar{s}}
$$

Therefore, the solution of Eq. (49) with the aid of Eq. (53) is given as

$$
\omega=-\frac{A i^{\prime}(\bar{s})+c B i^{\prime}(\bar{s})}{A i(\bar{s})+c B i(\bar{s})}
$$

Where $A i^{\prime}$ and $B i^{\prime}$ are the derivatives of the Airy functions. The initial condition gives the value of $c$ as

$$
c=-\frac{A i(-\bar{d})}{B i(-\bar{d})}
$$

The asymptotic bounded solutions for large values of $\bar{s}$ are given by

$$
\omega \sim \pm \sqrt{\bar{s}}+\frac{1}{4 \bar{s}}+\cdots \text { as } \bar{s} \rightarrow \infty
$$

Thus, there are two asymptotic solutions for large $\bar{s}$ for bounded $\omega$. The positive one, gives an increase of the temperature with time. That means that the solution follows the middle branch of the $S$ response curve. It can be shown, ${ }^{13}$ that this solution can only be reached for $d=2.338 \ldots$, which comes from the first zero of the Airy function $A i(-2.338 \ldots)=0$. If we note the asymptotic solution given by $\mathrm{Eq}$. (55), $\omega_{2}$, the stability of both solutions can be easily found by assuming a small perturbation of that solution of the form

$$
\omega=\omega_{\ell}+\hat{\omega} \exp (\nu \bar{s})
$$

Introduction of this relation into the Ricatti Eq. (49) shows that

$$
v=2 \omega_{e}
$$

Therefore, the positive asymptotic solution is unstable. This means that for values of $\bar{d}>$ $2.338 \ldots$, there is no longer a bounded solution for $\omega$, with $\omega \rightarrow \infty$ at a well defined time $\bar{s}_{I}$. To further follow the evolution of the temperature and radius of the particle, the analysis made in the supercritical regime, Eq. (41), will be adequate. On the other hand, for $d<$ $2.338 \ldots$ the solution initially tries to follow the middle branch. However, because this branch is unstable, $\omega$ reaches a maximum and then drops to negative values reaching the stable branch solution. This stable branch solution couples

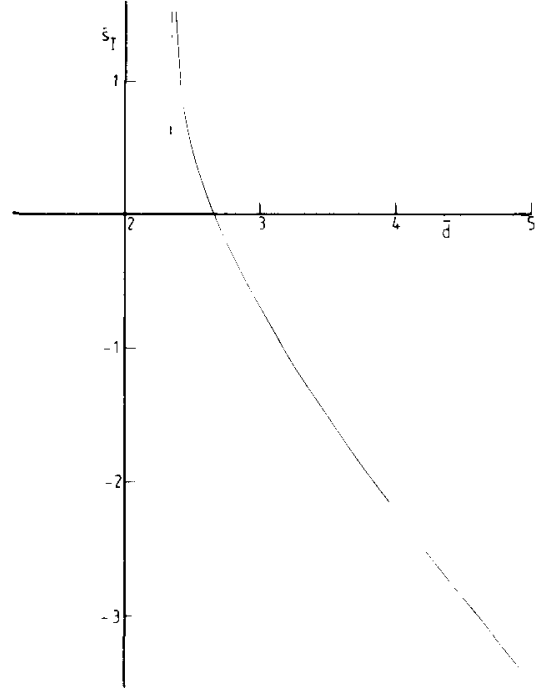

FIG, 4. Non-dimensional time at ignition $\bar{s}_{I}$ for critical regime as a function of the parameter $d$.

asymptotically with the subcritical regime analyzed earlier. More features of the solution to the Ricatti equation can be found elsewhere. ${ }^{13}$ Figure 4 shows the critical non-dimensional time $\bar{s}_{I}$ in function of $\bar{d}$.

\section{Concluding Remarks}

In this paper we have analyzed the behavior of spherical carbon particles suddenly immersed in a hot oxidizing atmosphere. Depending on the value of the surface Damköhler number, the particle undergoes different types of burning regimes. For values of the Damköhler of the order of unity, the particle is heated in a inert stage, reaching the ambient temperature asymptotically. There follows a transition stage where the particle radius does not experience any important change. Depending on the value of the Damköhler number, for the supercritical regime, there is a well defined time where the chemical reaction changes abruptly to be controlled by diffusion. At this time, the particle is consumed following the $R^{2}$ law. On the other hand, for the subcritical regime, the temperature of the particle reaches (without being consumed) a pseudo steady state followed by a very slow burning process, that does not follow the $R^{2}$ law for particle consumption. For Damköhler numbers very close to a critical one, there is a very slow transition stage, where the particle radius changes linearly with time until a condition is reached for supercritical behavior and there is a rapid increase in temperature. After this, the particle is con- 
sumed very rapidly, following the $R^{2}$ law. On the other hand, for subcritical behavior, the particle undergoes very slow burning throughout its life.

In this paper we have assumed a stagnant atmosphere. In this case, the assumption of a uniform temperature of the particle is fully justified because the thermal conductivity of carbon is greater than that of air. However, for turbulent flow, the particles follow only the large eddies. The influence of the smaller eddies on heat transfer is very important and has to be taken into account in this type of analysis. For rapid convective fluctuations, the temperature of the particles cannot be assumed to be uniform and the thermal diffusivity of the solid plays an important role in the burning behavior of the particle. This type of analysis is left for future work.

\section{Acknowledgments}

The participation of $\mathrm{C}$. Trevino in this work has been supported by a Grant of the Organization of American States (OAS). We thank the Ministerio de Educación y Ciencia of Spain, for supporting a short stay of C. Treviňo at the Escuela Técnica Superior de Ing. Aeronáuticos of the Universidad Politécnica de Madrid. We also acknowledge partial support of CONACYT of Mexico for this research.

\section{Nomenclature}

Ai Airy function

a Non-dimensional radius of particle defined in (10)

$B i \quad$ Airy function

$B$ Pre-exponential factor of the chemical reaction

$c_{p}$ Specific heat at constant pressure of the gas

$c$ Specific heat of the particle

d Non-dimensional parameter introduced in (42)

d Non-dimensional parameter defined in (51)

$D_{i} \quad$ Diffusion coefficient for specie $i$

$E$ Activation energy of the chemical reaction

I Non-dimensional thermal inertia of particle defined in $(15)$

Radiation factor

$\bar{J}$ Non-dimensional radiation parameter defined in (15)

$K$ Non-dimensional parameter introduced in (38)

Le Lewis number

$m$ Burning mass flow rate
$Q^{*} \quad$ Non-dimensional heat of reaction defined in (15)

$Q \quad$ Heat of reaction per unit mass of carbon consumed

Radius (coordinate)

$r \quad$ Reaction rate introduced in (1)

$\bar{R} \quad$ Universal gas constant

$R \quad$ Particle radius

$s \quad$ Non-dimensional time defined in (44)

$\bar{s} \quad$ Non-dimensional time defined in (50)

$T$ Temperature

$T_{\mathrm{s}} \quad$ Temperature at the gas-solid interface

$t_{g} \quad$ Characteristic time in the gas-phase

$t_{s} \quad$ Characteristic time in the solid-phase

$t$ Time

$u \quad$ Defined in (53)

$v \quad$ Eigenvalue introduced in (56)

$W_{i} \quad$ Molecular weight of species $i$

$Y_{i} \quad$ Mass concentration of species

$y$ Normalized $\mathrm{O}_{2}$ concentration defined in (10)

Z Zel'dovich number defined after (26)

Greek

$\alpha$ Non-dimensional temperture ratio defined in (15)

$\beta \quad$ Non-dimensional burning mass flow rate defined according to (15)

$\Delta \quad$ Damköhler number defined in (15)

$\delta \quad$ Reduced Damköhler number defined according to (26)

$\epsilon \quad$ Non-dimensionalized inverse of the activation energy def. (15)

$\phi_{1} \quad$ Non-dimensional variable defined in (43)

$\phi \quad$ Non-dimensional variable defined in (22)

$\lambda_{g}$ Thermal conductivity coefficient for the gas

$\lambda_{s} \quad$ Thermal conductivity coefficient for the solid

$\Lambda$ Non-dimensional parameter defined in $(50)$

$\bar{v} \quad$ Non-dimensional parameter defined in (15)

Gas density

$\rho_{s} \quad$ Solid density

$\sigma \quad$ Nondimensional time defined according to $(21)$

$\bar{o} \quad$ Non-dimensional time defined according to $(26)$

$\theta^{*}$ Critical non-dimensional temperature defined in (10)

$\theta_{c} \quad$ Critical non-dimensional temperature defined in (16)

$\theta$ Non-dimensional temperature defined in (10)

$7 \quad$ Non-dimensional time defined in (10)

$\omega$ Non-dimensional variable introduced in (50) 
Subscripts

c Critical

$d$ Diffusion control

e Steady state

g Gas

I Ignition

$i \quad$ Species

0 Initial

$\mathrm{O}_{2} \quad$ Oxygen

$s \quad$ Solid

$x \quad$ Ambient

\section{REFERENCES}

1. Matsui, K., Koyama, A. and Lehara, K.: Combust. and Flame, 25,57 (1975).

2. Tsuji, H. and Matsui, K.: Combust, and Flame, 26, $283(1976)$

3. Matsui, K., Tsujt, H. and Makino, A.: Combust. and Flame, 50, 107 (1983).
4. Adomeit, G., Hocks, W. and Henriksex, $K$., Combust, and Flame, 59, 107 (1985).

5. Adomeit, G. And Visser, W.: Twentieth Symposium (International) on Combustion, p. 1845 The Combustion Institute, 1984.

6. UBHAyakar, S. K.: Combust. and Flame, 26, 23 (1976).

7. Libby, P.A. and Blake, T.R.: Combust. and Flame, 36, 139 (1979).

8. Lisby, P.A.: Combust, and Flame, 38, 285 (1980).

9. Matalon, M.: Combust. Science and Technology, 24, 115 (1980)

10. LibBy, P.A. axd Blake, T.R.: Combust. and Flame, 41, 123 (1981).

11. Kassoy, D.R. and Libby, P.A.: Combust. and Flame, 48, 287 (1982).

12. Matalon, M.: SIAM J. Applied Math., 42, 787 (1982)

13. Kassoy, D.R. ANd Liñán, A.: Q. J. Mech. Applied Math., 31, 99 (1978) 